\title{
Superconducting crossed correlations in ferromagnets: implications for thermodynamics and quantum transport
}

\author{
R. Mélin \\ Centre de Recherches sur les Très Basses Températures (CRTBT) \\ CNRS, BP 166 X, 38042 Grenoble Cedex, France \\ and \\ Laboratoire de Physique, Ecole Normale Supérieure de Lyon \\ 46 Allée d'Italie, 69364 Lyon Cedex 07, France
}

(October 24, 2018)

\begin{abstract}
It is demonstrated that non local Cooper pairs can propagate in ferromagnetic electrodes having an opposite spin orientation. In the presence of such crossed correlations, the superconducting gap is found to depend explicitly on the relative orientation of the ferromagnetic electrodes. Non local Cooper pairs can in principle be probed with dc-transport. With two ferromagnetic electrodes, we propose a "quantum switch" that can be used to detect correlated pairs of electrons. With three or more ferromagnetic electrodes, the Cooper pair-like state is a linear superposition of Cooper pairs which could be detected in dc-transport. The effect also induces an enhancement of the ferromagnetic proximity effect on the basis of crossed superconducting correlations propagating along domain walls.

PACS numbers: 74.50.+r, 03.67.-a, 74.80.Fp
\end{abstract}

Ferromagnetism and superconductivity are antagonist correlated states of matter. In ferromagnetism, one spin population is favored because of spin symmetry breaking, while in $s$-wave superconductivity, electrons with an opposite spin are bound into Cooper pairs because of the attractive electron-electron interaction. It has been a long standing problem to determine to what extend these two orders can coexist in the same system. As first proposed 40 years ago by Anderson and Suhl, the coexistence is possible if the ferromagnet acquires a cryptomagnetic [1, or cryptomagnetic-like [2] domain structure. On the other hand, in superconductor / ferromagnet heterostructures, a Cooper pair penetrating into a single domain ferromagnet acquires a finite kinetic energy due to the coupling to the exchange field. This results in a spatial oscillation of the induced superconducting order parameter 3 6], giving rise to the so-called $\pi$-state, which has been probed recently in two experiments [7, 8 ]. In this Letter, we consider Cooper pair penetration in a multi domain ferromagnet. It has been already shown theoretically that crossed Andreev reflections can arise in a heterostructure in which two ferromagnets with an opposite spin orientation are connected to a superconductor [9]. Such Andreev reflections do not exist when a single domain ferromagnet is in contact with a superconductor 10 12]. We demonstrate here that quasi long range superconducting correlations can propagate in two magnetic domains with an opposite magnetization. These correlations correspond to non local Cooper pair-like objects in which the spin-up (down) electron propagates in a spin-up (down) ferromagnetic domain.

This implies several consequences that may be tested in future experiments. First, considering the problem from the point of view of a superconductor order parameter coupled to a ferromagnetic environment, we show that the transition temperature of the superconductor depends explicitly on the relative spin orientations of the electrodes. The superconducting gap is smaller when the electrodes are misoriented.

The second implication of the model is that ferromagnetic domain walls can propagate superconducting crossed correlations, in which the two electrons making a Cooper pair reside in neighboring magnetic domains. This may apply to explain the enhancement of the proximity effect observed in ferromagnet / superconductor heterostructures 13,16 .

The third implication of the model is related to the production and measurement of linear superpositions of non local Cooper pairs. It was stressed by Einstein, Podolsky and Rosen (EPR) in 1935 [17] that non locality was a deep feature of quantum mechanics. Non locality 18] has been probed experimentally with photons $[19,20]$. Condensed matter systems provide maybe the opportunity to fabricate entangled states with electrons, being massive particles, and to fabricate quantum bits, which would be the building blocks of a quantum computer 21 24 . Two proposals have been made recently: one is based on tunneling in a double quantum dot [25], and the other is based on noise correlations of Cooper pairs emitted in a beam splitter [26]. We show that superconducting crossed correlations in ferromagnets provide a possibility to manipulate linear superpositions of Cooper pairs. With two ferromagnetic electrodes, we propose a "quantum switch" device that can be used to detect correlated pairs of electrons. Linear superpositions can be obtained with three or more ferromagnetic electrodes, and can be probed in a dc-transport.

Let us now consider a microscopic model in which a superconductor is connected to external electrodes. The superconductor is represented by the single site ef- 
fective Nambu Green's function 27] $\hat{g}^{R, A}(\omega)=g(\omega \pm$ $i \eta) \hat{I}+f(\omega \pm i \eta) \hat{\sigma}^{x}$, with $g(\omega)=-\pi \rho_{N} \omega / \sqrt{\Delta^{2}-\omega^{2}}$, and $f(\omega)=\pi \rho_{N} \Delta / \sqrt{\Delta^{2}-\omega^{2}}$, and where $\rho_{N}$, having the dimension of an inverse energy, is the normal state density of states, $\hat{I}$ is the $2 \times 2$ identity matrix, and $\hat{\sigma}^{x}$ a Pauli matrix. We assume that $N$ ferromagnetic electrodes are in contact with the superconductor (see Fig. 11 (a)), with a hopping Hamiltonian $W=\sum_{k=1}^{N} t_{x, \alpha_{k}}\left[c_{\alpha_{k}}^{+} c_{x}+c_{x}^{+} c_{\alpha_{k}}\right]$. The electrode $k$ having a spin polarization $P_{k}=\left(\rho_{k, \uparrow}-\right.$ $\left.\rho_{k, \downarrow}\right) /\left(\rho_{k, \uparrow}+\rho_{k, \downarrow}\right)$ is represented by the Green's function $\hat{g}_{k}^{A, R}= \pm i \pi\left[\rho_{k, \uparrow}\left(\hat{I}+\hat{\sigma}^{z}\right) / 2+\rho_{k, \downarrow}\left(\hat{I}-\hat{\sigma}^{z}\right) / 2\right]$. We use a perturbation theory in the tunnel amplitude $W$, which we sum up to infinite order 27,28]. The Dyson equation takes the form

$$
\hat{G}_{x, x}^{R, A}=\left[\hat{I}-\sum_{k=1}^{N} \hat{g}_{x, x}^{R, A} \hat{t}_{x, \alpha_{k}} \hat{g}_{\alpha_{k}, \alpha_{k}}^{R, A} \hat{t}_{\alpha_{k}, x}\right]^{-1} \hat{g}_{x, x}^{R, A},
$$

where $\hat{t}_{\alpha_{k}, x}$ is the Nambu representation of the tunnel matrix element: $\hat{t}_{\alpha_{k}, x}=t_{\alpha_{k}, x} \hat{\sigma}^{z}$. The relevant parameters appear to be the spectral line-width associated to spin- $\sigma$ electrons: $\Gamma_{\sigma}=\sum_{k=1}^{N} \Gamma_{k, \sigma}$, with $\Gamma_{k, \sigma}=$ $\left(t_{\alpha_{k}, x}\right)^{2} \rho_{k, \sigma}$. Solving Eq. 目leads to

$$
\begin{aligned}
\hat{G}_{x, x}^{A} & =\frac{1}{\mathcal{D}}\left\{g \hat{I}+f \hat{\sigma}^{x}\right. \\
& \left.+i \pi\left(f^{2}-g^{2}\right)\left[\frac{\Gamma_{\downarrow}}{2}\left(\hat{I}+\hat{\sigma}^{z}\right)+\frac{\Gamma_{\uparrow}}{2}\left(\hat{I}-\hat{\sigma}^{z}\right)\right]\right\},
\end{aligned}
$$

with $\mathcal{D}=1-i \pi g\left(\Gamma_{\uparrow}+\Gamma_{\downarrow}\right)+\pi^{2}\left(f^{2}-g^{2}\right) \Gamma_{\uparrow} \Gamma_{\downarrow}$. To calculate the superconducting order parameter, we need to solve the Dyson-Keldysh equation $\hat{G}^{+-}=\left(\hat{I}+\hat{G}^{R} \otimes \hat{W}\right) \otimes$ $\hat{g}^{+-} \otimes\left(\hat{I}+\hat{W} \otimes \hat{G}^{A}\right)$, where the convolution includes a sum over the labels $x$ and $\alpha_{k}$. Noting $X_{\sigma}=\left(1-i \pi g \Gamma_{-\sigma}\right) / \mathcal{D}$, $Y_{\sigma}=i \pi f \Gamma_{\sigma} / \mathcal{D}$, and using Eq. 2, we obtain the exact expression of the Nambu component of the Keldysh Green's function:

$$
\begin{aligned}
{\left[G_{x, x}^{+-}\right]_{2,1} } & =2 i \pi n_{F}(\omega) \times \\
& \left\{\rho_{g}\left(X_{\uparrow} \bar{Y}_{\uparrow}+Y_{\downarrow} \bar{X}_{\downarrow}\right)+\rho_{f}\left(X_{\uparrow} \bar{X}_{\downarrow}+Y_{\downarrow} \bar{Y}_{\uparrow}\right)\right. \\
& \left.+\frac{1}{\pi^{2} \Gamma^{\downarrow}} \bar{Y}^{\downarrow}\left(X^{\uparrow}-1\right)+\frac{1}{\pi^{2} \Gamma^{\uparrow}} Y^{\uparrow}\left(\bar{X}^{\downarrow}-1\right)\right\},
\end{aligned}
$$

where $n_{F}(\omega)$ is the Fermi distribution, and we used the notation $\hat{\rho}=\rho_{g} \hat{I}+\rho_{f} \hat{\sigma}^{x}=\operatorname{Im}\left[\hat{g^{A}}\right] / \pi$. The superconducting gap is obtained by imposing the self-consistent equation $\Delta=U \int_{-\infty}^{+\infty} d \omega /(2 i \pi)\left[\hat{G}^{+-}(\omega)\right]_{2,1}$ [29], with $U$ the microscopic attractive interaction. The dominant contribution arises from the large- $|\omega|$ behavior and we obtain a BCS-type relation:

$$
\Delta=D \exp \left[-\frac{1}{\rho_{N} U}\left(1+\pi \rho_{N} \Gamma_{\uparrow}\right)\left(1+\pi \rho_{N} \Gamma_{\downarrow}\right)\right],
$$

with $D$ the bandwidth of the superconductor. As an example, we consider a coupling to two ferromagnets.
With a parallel alignment of the magnetization in the electrodes, we have $\Gamma_{\uparrow}=2 \gamma$ and $\Gamma_{\downarrow}=0$. With an antiparallel alignment, we have $\Gamma_{\uparrow}=\Gamma_{\downarrow}=\gamma$. The ratio of the two gaps is found to be

$$
\frac{\Delta_{A P}}{\Delta_{P}}=\exp \left(-\frac{\pi^{2} \rho_{N} \gamma^{2}}{U}\right)
$$

which shows that the spin polarized environment generates a reduction of the superconducting gap that depends explicitly on the spin orientation of the environment. The transition temperature of the superconductor is larger if the electrodes are in an antiparallel alignment. This behavior should be contrasted with another model proposed recently 30 .

(a)

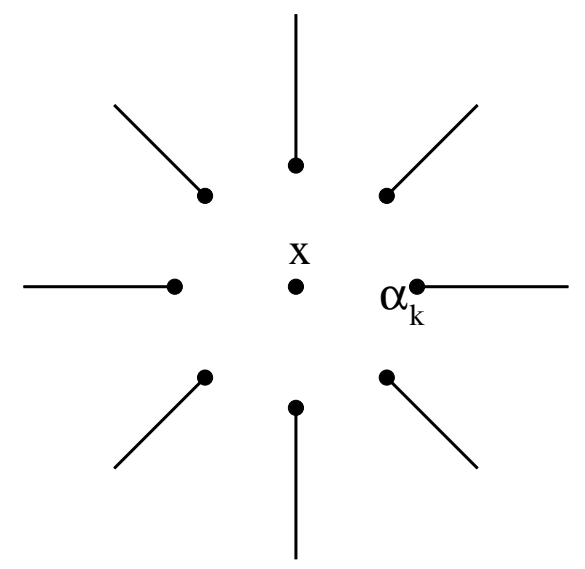

(b)

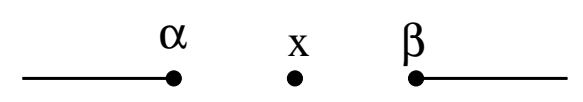

FIG. 1. Schematic representation of the models. In (a): the model with a coupling of the superconducting site $x$ to $N$ ferromagnetic electrodes. In (b), the model with a coupling to two ferromagnetic electrodes.

As we show now, the gap variation Eq. 同 is due to the possibility that superconducting pairs can delocalize in the ferromagnetic electrodes having an opposite spin orientation. Let us consider the problem with two electrodes only. The two electrodes are labeled by the Greek indices $\alpha_{1}=\alpha$ and $\alpha_{2}=\beta$. We use Eq. 3 to calculate exactly the crossed Keldysh Green's functions:

$$
\begin{aligned}
& {\left[G_{\alpha, \beta}^{+-}\right]_{2,1}=i\left\langle c_{\beta, \uparrow}^{+} c_{\alpha, \downarrow}^{+}\right\rangle=\pi^{2} t_{\alpha} t_{\beta} \rho_{\alpha, \downarrow} \rho_{\beta, \uparrow}\left[G_{x, x}^{+-}\right]_{2,1}} \\
& {\left[G_{\alpha, \beta}^{+-}\right]_{1,2}=i\left\langle c_{\beta, \downarrow} c_{\alpha, \uparrow}\right\rangle=\pi^{2} t_{\alpha} t_{\beta} \rho_{\alpha, \uparrow} \rho_{\beta, \downarrow}\left[G_{x, x}^{+-}\right]_{1,2},}
\end{aligned}
$$

with $\left[\hat{G}_{x, x}^{+-}\right]_{2,1}=\overline{\left[\hat{G}_{x, x}^{+-}\right]_{1,2}}$ given by Eq. 3. The density of states prefactors in Eqs. 6 , 7 appear to be a direct consequence of the Pauli exclusion principle. To show this, we consider Eqs. 6, 7] in the limit of fully polarized ferromagnets. In the parallel alignment $\left(\rho_{\alpha, \uparrow}=\rho_{\beta, \uparrow}=1\right.$, 
$\left.\rho_{\alpha, \downarrow}=\rho_{\beta, \downarrow}=0\right)$, all pair correlations are vanishing: $\left\langle c_{\beta, \uparrow}^{+} c_{\alpha, \downarrow}^{+}\right\rangle=\left\langle c_{\beta, \downarrow} c_{\alpha, \uparrow}\right\rangle=0$. This is fully expected because one cannot add or destroy a spin-down electron in the presence of a spin-up band only. For the same reason, one has $\left\langle c_{\beta, \uparrow}^{+} c_{\alpha, \downarrow}^{+}\right\rangle=0$ in the antiparallel alignment $\left(\rho_{\alpha, \uparrow}=\rho_{\beta, \downarrow}=1, \rho_{\alpha, \downarrow}=\rho_{\beta, \uparrow}=0\right)$. The remaining non vanishing crossed correlations are $\left\langle c_{\beta, \downarrow} c_{\alpha, \uparrow}\right\rangle$ and $\left\langle c_{\beta, \downarrow}^{+} c_{\alpha, \uparrow}^{+}\right\rangle$. This shows the possibility to generate superconducting crossed correlations in two ferromagnets with an opposite magnetization. To characterize the propagation of crossed correlations, we calculate the Gorkov function $\hat{G}_{i, j}^{+-}$, with $i$ and $j$ two sites in the ferromagnetic electrodes $\alpha$ and $\beta$ such that $x_{i}=-x_{j}$. Assuming that the ferromagnetic electrodes behave like a three dimensional metal, we find

$$
\left[\hat{G}_{i, j}^{+-}\right]_{1,2} \sim \frac{1}{\left|x_{i}\right|} \pi^{2} t_{\alpha} t_{\beta} \rho_{\alpha, \uparrow} \rho_{\beta, \downarrow}\left[\hat{G}_{x, x}^{+-}\right]_{1,2} .
$$

By comparison, there is a density of states prefactor $\rho_{\alpha, \uparrow} \rho_{\alpha, \downarrow}$ in the local superconducting correlation in electrode $\alpha$. As a consequence, in strongly spin polarized ferromagnets, superconducting crossed correlations can propagate while ordinary superconducting correlations cannot propagate. It is well known that there is an oscillating induced order parameter associated to Cooper pair penetration in partially spin polarized ferromagnets [2 6]. There are no such oscillations in the case of crossed correlations because Cooper pairs do not acquire a center of mass momentum when entering the ferromagnetic electrodes.

(a)

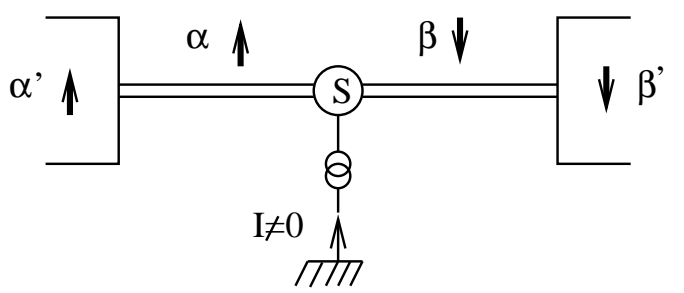

(b)

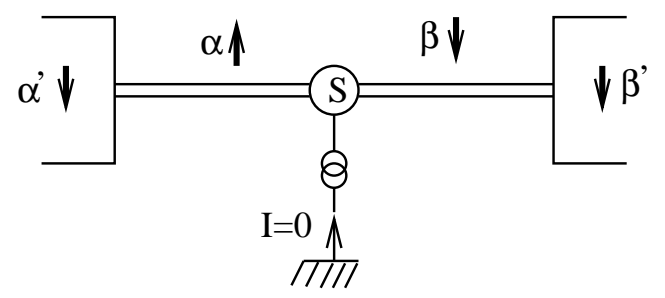

FIG. 2. Schematic representation of the quantum switch to probe correlated pairs of electrons. A current source is connected to the superconductor. In (a), there is a finite current flowing. In (b), there is no current flowing.

The model can be considered from the point of view of propagation of cross-correlated Cooper pairs along domain walls in a multi domain ferromagnet. Such crossed correlations can generate an enhancement of the ferromagnetic superconducting proximity effect, which is not against recent experiments in ferromagnet / superconductor heterostructures 13 15. Another proposal based on spin accumulation has been made recently [16], but appears to be incompatible with some experiments [14]. Our scenario and the spin accumulation picture both contribute to the same effect, but in a different situation: crossed correlations can propagate only in multi domain ferromagnets, while the spin accumulation mechanism is valid even with single domain ferromagnets.

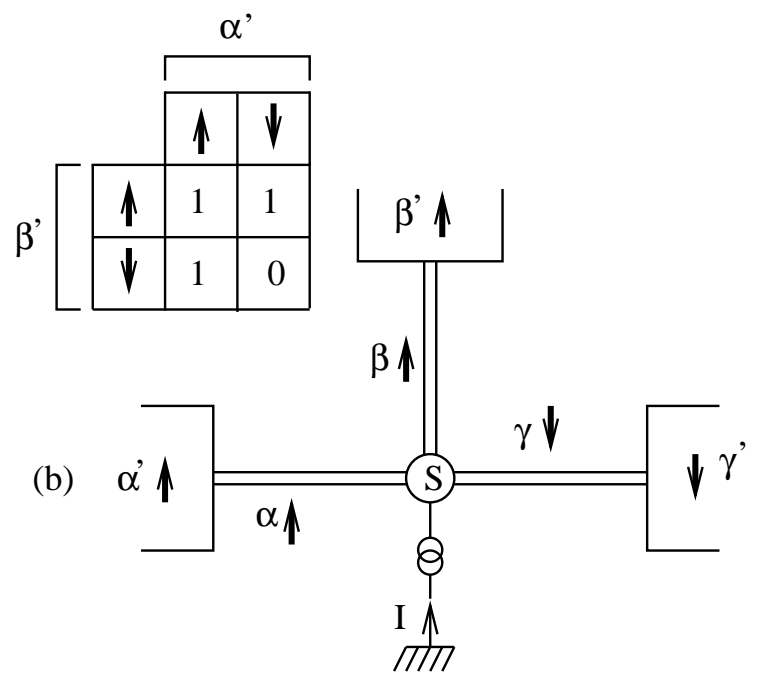

FIG. 3. Schematic representation of the three-terminal device used to probe linear superposition of Cooper pairs. The insert shows the presence/absence of a current flowing into the superconductor as a function of the spin orientation in the ferromagnetic reservoirs.

Now we show that superconducting crossed correlations can be used to produce correlated pairs of electrons. Let us consider two ferromagnets $\alpha$ and $\beta$ in contact with a superconductor. The cross-correlated degrees of freedom are represented by the Cooper pair-like wave function $|\psi\rangle=\left[u_{0}+v_{0} c_{\alpha, \uparrow}^{+} c_{\beta, \downarrow}^{+}\right]|0\rangle$, with $u_{0}$ and $v_{0}$ the BCS coherence factors. Let us consider two additional ferromagnetic electrodes $\alpha^{\prime}$ and $\beta^{\prime}$ having a spin orientation $\Sigma_{\alpha^{\prime}}$ and $\Sigma_{\beta^{\prime}}$ connected to the electrodes $\alpha$ and $\beta$ (see Fig. 2). The electrodes $\alpha^{\prime}$ and $\beta^{\prime}$ are considered to be reservoirs in which all inelastic processes take place. For the sake of obtaining the basic physics of such systems, we restrict ourselves to fully polarized ferromagnets and high transparency contacts 31. If $\Sigma_{\alpha^{\prime}}=\uparrow, \Sigma_{\beta^{\prime}}=\downarrow$, the correlated pair can be transmitted into the reservoirs $\alpha^{\prime}$ and $\beta^{\prime}$ and a finite current is flowing into the superconductor (see Fig. 2 (a)). If $\Sigma_{\alpha^{\prime}}=\Sigma_{\beta^{\prime}}=\downarrow$, the spin-up electron making the correlated state is backscattered at the interface with the spin-down ferromagnet $\alpha^{\prime}$. Coming back onto the superconductor interface it undergoes a crossed Andreev reflection [9] in which a Cooper is formed in the superconductor and a spin-down hole is transferred into electrode $\beta$. The whole process does not carry elec- 
trical charge: there is no current transmitted into the superconductor (see Fig. 2 (b)). The "quantum switch" device on Fig. 2 2 can therefore be used to produce and detect correlated pairs of electrons with dc-transport.

Now we discuss the production of linear superpositions in a three-terminal device (see Fig. 3). The three ferromagnetic electrodes are labeled by the indices $\alpha_{1}=\alpha, \alpha_{2}=\beta$ and $\alpha_{3}=\gamma$. With fully polarized ferromagnets having a spin orientation $\sigma_{\alpha}=\sigma_{\beta}=\uparrow$, $\sigma_{\gamma}=\downarrow$, the exact form of the crossed-correlations is given by $\left[\hat{G}_{\alpha(\beta), \gamma}^{+-}\right]_{1,2}=\pi^{2} t_{\alpha(\beta)} t_{\gamma}\left[\hat{G}_{x, x}^{+-}\right]_{1,2}$. This means that Cooper pairs can delocalize over several electrodes. The corresponding wave function is a linear superposition of Cooper pairs $|\psi\rangle=\lambda_{\alpha}\left[u_{0}+v_{0} c_{\alpha, \uparrow}^{+} c_{\gamma, \downarrow}^{+}\right]|0\rangle+$ $\lambda_{\beta}\left[u_{0}+v_{0} c_{\beta, \uparrow}^{+} c_{\gamma, \downarrow}^{+}\right]|0\rangle$. The coefficients $\lambda_{\alpha}$ and $\lambda_{\beta}$ are such that the Cooper pair wave function contains the same pair correlations as the Gorkov function: $\left\langle c_{\beta, \uparrow}^{+} c_{\gamma_{\downarrow}}^{+}\right\rangle /\left\langle c_{\alpha, \uparrow}^{+} c_{\gamma_{\downarrow}}^{+}\right\rangle=\lambda_{\beta} / \lambda_{\alpha}=t_{\beta} / t_{\alpha}$, from what we deduce $\lambda_{\alpha(\beta)}=t_{\alpha(\beta)} / \sqrt{t_{\alpha}^{2}+t_{\beta}^{2}+2 u_{0}^{2} t_{\alpha} t_{\beta}}$. As a direct consequence of the linear superposition, the current flowing into the superconductor is vanishing if $\Sigma_{\alpha^{\prime}}=$ $\Sigma_{\beta^{\prime}}=\downarrow$ and finite in the three other spin orientations (see Fig. 3). Now the linear superposition associated to the electrodes magnetization $\sigma_{\alpha}=\uparrow, \sigma_{\beta}=\sigma_{\gamma}=\downarrow$ is $|\psi\rangle=\lambda_{\beta}^{\prime}\left[u_{0}+v_{0} c_{\alpha, \uparrow}^{+} c_{\beta, \downarrow}^{+}\right]|0\rangle+\lambda_{\gamma}^{\prime}\left[u_{0}+v_{0} c_{\alpha, \uparrow}^{+} c_{\gamma, \downarrow}^{+}\right]|0\rangle$, with $\lambda_{\beta(\gamma)}^{\prime}=t_{\beta(\gamma)} / \sqrt{t_{\beta}^{2}+t_{\gamma}^{2}+2 u_{0}^{2} t_{\beta} t_{\gamma}}$. The current flowing into the superconductor is vanishing in the two spin orientations $\Sigma_{\alpha^{\prime}}=\downarrow, \Sigma_{\beta^{\prime}}=\uparrow, \downarrow$ and finite otherwise. Therefore, a dc-current measurement can make the distinction between the linear superpositions associated to the spin orientations $\sigma_{\alpha}=\sigma_{\beta}=\uparrow, \sigma_{\gamma}=\downarrow$ and $\sigma_{\alpha}=\uparrow$, $\sigma_{\beta}=\sigma_{\gamma}=\downarrow$.

To conclude, we have shown that quasi long range superconducting crossed correlations can propagate in ferromagnets having an opposite spin orientation. The superconducting crossed correlations are much stronger than the local ones. Such crossed correlations can propagate along ferromagnetic domain walls, and contribute to an enhancement of the ferromagnetic proximity effect, which may apply to recent experiments 13 15. The superconducting gap depends explicitly on the spin orientation of the ferromagnetic electrodes, which could be used as an experimental probe of superconducting crossed correlations. We have shown that crossed correlations can be used to produce correlated pairs of electrons and linear superpositions of correlated pairs. Such states can in principle be detected with dc-transport. The microscopic calculation of the current will be the subject of a future work.

The author acknowledges unvaluable discussions with P. Degiovanni, D. Feinberg and M. Giroud.
[1] P. Anderson and H. Suhl, Phys. Rev. 116, 6739 (1959).

[2] A. Buzdin and L. Bulaevskii, JETP 67, 576 (1988).

[3] P. Fulde and A. Ferrel, Phys. Rev. 135, A550 (1964).

[4] A. Larkin and Y. Ovchinnikov, Sov. Phys. JETP 20, 762 (1965).

[5] M. A. Clogston, Phys. Rev. Lett. 9, 266 (1962).

[6] E.A. Demler, G.B. Arnold and M.R. Beasley, Phys. Rev. B 55, 15174 (1997).

[7] V.V. Ryazanov et al., arXiv:cond-mat/0008364

[8] T. Kontos, M. Aprili, J. Lesueur and X. Grison, arXiv:cond-mat/0009192.

[9] G. Deutscher and D. Feinberg, App. Phys. Lett. 76, 487 (2000).

[10] M.J.M. de Jong and C.W.J. Beenakker, Phys. Rev. Lett. 74, 1657 (1995).

[11] R.J. Soulen et al., Science 282, 85 (1998).

[12] S.K. Upadhyay et al., Phys. Rev. Lett. 81, 3247 (1998).

[13] M. Giroud et al., Phys. Rev. B 58, R11872 (1998).

[14] V.T. Petrashov, I.A. Sosnin, and C. Troadec, arXiv:condmat/0007278

[15] J. Aumentado and V. Chandrasekhar, arXiv:condmat/0007433

[16] W. Belzig, A. Brataas, Yu. V. Nazarov, and G.E. Bauer, arXiv:cond-mat/0005188.

[17] A. Einstein, B. Podolsky, and N. Rosen, Phys. Rev. 47, 777 (1935).

[18] J.S. Bell, Physics (N.Y.) 1, 195 (1965).

[19] A. Aspect, P. Grangier, and G. Roger, Phys. Rev. Lett. 47, 460 (1981); A. Aspect, J. Dalibard, and G. Roger, ibid. 49, 1904 (1982).

[20] P.G. Kwiat et al., Phys. Rev. Lett. 75, 4337 (1995).

[21] P.W. Schor, in Proc. 35th Symposium on the Foundations of Computer Science (IEEE Computer Society Press), 124 (1994).

[22] L.K. Grover, Phys. Rev. Lett. 79, 325 (1997).

[23] A. Steane, Rep. Prog. Phys. 61, 117 (1998).

[24] Y. Nakamura, Yu. A. Pashkin, and J.S. Tsai, Nature 398, 786 (1999).

[25] D. Loss and E.V. Sukhorukov, Phys. Rev. Lett. 84, 1035 (2000).

[26] G.B. Lesovik, T. Martin, and G. Blatter, arXiv:condmat/0009193

[27] J.C. Cuevas, A. Martin-Rodero, and A. Levy Yeyati, Phys. Rev. B 54, 7366 (1996).

[28] C. Caroli, R. Combescot, P. Nozières, and D. SaintJames, J. Phys. C 5, 21 (1972).

[29] A. Martin-Rodeiro, F.J. Garcia-Vidal, and A. LevyYeyati, Phys. Rev. Lett. 72, 554 (1994).

[30] I. Baladie, A. Buzdin, N. Ryzhanova, A. Vedyayev, Phys. Rev. B 63, 054518 (2001).

[31] The Keldysh formalism can be used to calculate the current-voltage characteristics in the presence of arbitrary spin polarizations and contact transparencies $[R$. Mélin, unpublished]. 